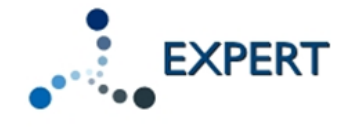

Project funded by the People Programme (Marie Curie Actions) of the European Union's Seventh Framework Programme FP7/2007-2013/ under REA grant agreement no. 317471 .

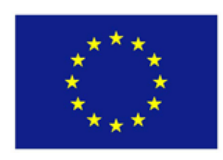

Project reference: 317471

Project full title: EXPloiting Empirical appRoaches to Translation

\title{
D2.1: User Requirement Analysis
}

Authors: Anna Zaretskaya (UMA),

Contributors: Miriam Seghiri (UMA), Gloria Corpas (UMA), Santanu Pal (USAAR), Varvara Logacheva (USFD)

Document Number: EXPERT_D2.1_20150210

Distribution Level: Public

Contractual Date of Delivery: 31.07.14

Actual Date of Delivery: 10.02.15

Contributing to the Deliverable: WP2

WP Task Responsible: UMA

EC Project Officer: Concepcion Perez-Camaras 
Contents:

1. User Needs Identification for Translation Systems 2

2. Purpose of the Survey and Research Questions 4

3. Translation Technologies and Current User Needs 5

4. Questionnaire Design and Implementation 6

5. Participants and their Profile 8

$\begin{array}{ll}\text { 6. Use of Tools } & 10\end{array}$

$\begin{array}{ll}\text { 7. Machine Translation: Practices and Attitudes } & 14\end{array}$

8. Translation Memory: Working Practices and Offered Functionalities 17

9. Textual Corpora and Related Tools 20

$\begin{array}{ll}\text { 10. Conclusions } & 23\end{array}$

$\begin{array}{ll}\text { 11. References } & 25\end{array}$ 


\section{User Needs Identification for Translation Systems}

Understanding user needs and requirements is a crucial step for designing interactive computer systems. User-centred design can increase productivity, enhance work quality, reduce support costs, and increase general user satisfaction. This concerns translation systems as well. The invasion of technology in the field of translation forces professionals to use sometimes highly complex computer programs and resources in order to keep up with the industry requirements. Even though there exist a large variety of such computer programs on the market, previous surveys on translation technologies (Lagoudaki, 2006; Blacafort et al., 2011; TradOnline, 2010; Torres Domínguez, 2012) show that not all of the existing technologies were successfully adopted by professionals. One of the possible reasons for this is that the programs were developed without taking into account translators' real needs.

User requirement analysis is not a simple process. Some of the most common obstacles are the following:

- When there is a new type of system to be developed, it is a logical step to ask the target users about how they would like the system to be. However, no system of this type exists yet, so the users cannot base their opinions on experience, and therefore it is difficult for them to decide which characteristics they want the system to have.

- Users and designers often think within traditional boundaries and leave no place for innovation.

- It is not clear how exactly these requirements must be represented so that the system designers can incorporate them in the development process.

- Finally, it is also necessary to decide on the best way to identify these requirements, which depend on the user profile as well as on specific aspects of the system to be developed.

One of the common methods for user requirements identification is user surveys. It consists in administering a set of written questions to a sample population of users (Maguire \& Bevan, 2002: 137). One of the biggest advantages of this method is that it allows to reach a large population of users with minimal costs. Furthermore, surveys are normally composed of both closed and open types of questions, allowing to obtain both quantitative and qualitative data in large quantities.

Focus groups are another way of gathering information on user needs. They bring together a group of stakeholders in a format of discussion group. During these discussions, each participant's actions can stimulate ideas in other group members and as the discussion goes on, the collective view becomes established which is broader and more objective than the individual parts (Langford \& McDonagh, 2003). 
One more method of identifying user requirements is interviewing, where users are questioned in a semi-structured way, i.e. the interview contains some fixed questions but also allows the interviewees to expand their answers in a free manner (Courage \& Baxter, 2005: pp. 246-312). Interviews allow to collect very rich, detailed data thus providing a holistic view of the picture. However, they are not suitable for gathering information from a large sample of users.

Scenarios of use provide detailed information how the users will carry out their tasks and interact with the future system in a real working setting. They are built specifically for understanding the users' working practices and possible related requirements and for providing examples for future use and probably information on task completion time (Maguire \& Bevan, 2002: 137).

Finally, evaluating existing or competitor systems can provide information on whether the existing systems meet user requirements and to which extent, and help identify existing usability problems that should be avoided in future systems. On the other hand, it can also indicate the features that are considered useful in existing systems and that should be included in the future systems as well.

Other techniques for obtaining feedback from users, such as brainstorming and card sorting, are described more in details by Courage and Baxter (2005), and Maguire and Bevan (2002). All of these methods are suitable for different purposes and development stages, as some of them (for instance, interviews) are good methods for developing a general picture of initial set of requirements, while others (like card sorting) are more useful for validating an existing system prototype.

In translation technologies, some specific techniques are used to gather users' feedback by registering interaction between users and translation systems. These techniques are mostly used for improvement of already existing systems. Thus, the most popular source of user attitude towards output of machine translation (MT) systems is the post-editing process. Its outcome can be extremely useful for MT systems developers as they provide the real-world user feedback. This feedback is particularly valuable because it appears as an outcome of natural work process, i.e. the data generation is done without any additional arrangements and expenses and without disturbing the translator's natural workflow. Even if the translators do not evaluate the translation quality explicitly, the evaluation can be inferred, for instance, by the amount of editing performed or by the amount of accepted or rejected sentences. Post-editing can be used to extract different types of human feedback of different types and levels of granularity:

- binary quality score (good/bad);

- fine-grained quality score (the percentage of changed words);

- correct translations of incorrectly translated words or phrases;

- time spent on correction of different segments. 
All this information can be fed back to MT systems in order to improve them. The online re-training of MT systems is an especially promising direction, as it allows improving a system continuously while a translator is working and dynamically adapt to the domain of a current document. In addition, the analysis of post-editions can help identify potentially wrong segments in automatic translations.

It is also worth mentioning that post-editing of automatic translations is not the only way of user interaction with MT systems. The interactive machine translation (IMT) (Ortiz-Martínez et al., 2010) architecture has been designed particularly to suit the human-in-the-loop scenario, although the case studies show that some users find the work with IMT systems ineffective (Alabau et al., 2012). Another type of scenario allows a user to compose a sentence from translation options generated by an MT system (Koehn \& Haddow, 2009). These technologies have not been implemented in the most popular CAT systems yet. However, they need to be examined in order to understand if any of them can be accepted by the translation community as alternative ways to gather user feedback for identifying user requirements for these tools.

\section{Purpose of the Survey and Research Questions}

In our research we opted for the survey method to gather information on user needs for various reasons. First of all, our task consisted in covering a broad range of different types of computer programs and resources, including, first of all, machine translation and translation memory systems, but also corpora building tools terminology management systems and others. The survey format allowed us to obtain information about various aspects of all these tools without being limited to one specific type of systems. Furthermore, we aimed to reach different user groups as each of groups has a different profile and therefore different requirements. For instance, translators who work in-house in a translation company supposedly have a workflow and working habits which are different from those of freelance translators. Finally, as has been mentioned before, the survey method allowed us to obtain and analyse both quantitative and qualitative data, which can contribute to answering previously formulated research questions as well as give new ideas about what professional translators actually need.

In this report we provide preliminary results of the online survey for professional translators. Its main goal was to identify

1) current working practices of professional translators, i.e. which tools and resources they use and how they do it;

2) degree of satisfaction with these technologies concerning the quality of output, learning curve, offered functionalities, productivity and income increase;

3) levels of awareness of different types of technologies available; 
4) possible reasons for low usage rate for different tools and missed opportunities for reaching potential users;

5) overall attitude towards current technology-related industry trends;

6 ) ways that can lead to creating future systems and to expanding and improving existing tools.

The results discussed here correspond to the first, descriptive analysis of the data and intend to shed light on the participants' profile, respondent groups regarding their employment type and experience, familiarity and usage rates of various types of technologies, and attitudes towards their features and functionalities they offer.

\section{Translation Technologies and Current User Needs}

From a translator's point of view, translation tools are computer software that aims to facilitate the work of translators, make the project delivery faster and easier, save translators' time by solving easier tasks in an automatised way and allow them to concentrate on more challenging and creative parts of the translation process, and finally, to increase their income (Bowker and Corpas Pastor, 2014/In press). In practice, the amount of tools available also makes translator's life difficult, as they have to decide which tools are useful for them and how to integrate them in their workflow. Thus, machine translation (MT) services available nowadays not only for translators but also for common users evoke contradictory attitudes among translators. On one hand they are cheap and easy to use, and therefore can provide a fast draft translation. On the other hand, the quality of translation is not satisfactory enough for all domains and languages even as a draft, so many translators find them useless for their job and prefer to make translations from scratch. In addition, there is an increasing concern related to the security of the information translated on the Web, and many translators who do like working with MT are imposed to sign confidentiality agreements with their clients for not using any such service.

The main type of technology that prevails on today's market are translation memory systems. Since their appearance on the market, these tools are positively accepted by the majority of translators as they seem to serve the purpose of time and cost saving. Indeed, now the translator does not have to spend time typing the same segments of text that were already translated before. However, developers of these tools keep adding more and more features and functionalities, which are often too complex and require much time and effort to learn. Therefore, adopting these tools becomes a challenge for translators, which is we need to investigate which of the features translators actually use and find helpful. Such technologies as terminology extractors, tools for compiling corpora, and especially automatic translation systems are already integrated in some translation software (for instance, the terminology system SDL 
Multiterm ${ }^{1}$ in SDL Trados Studio ${ }^{2}$, the corpora building system LiveDocs in MemoQ ${ }^{3}$, among others), and additionally they are also available as standalone programs that can be used aside when there is such need. It is of interest to find out how translators prefer to work with these tools, whether they mostly use integrated or standalone systems, and what degree of flexibility should developers allow in this relation to satisfy users with different tastes and preferences.

Another recent industry development is linked to the increasing amounts of translationrelated resources available online, such as termbanks and translation memory repositories (like MyMemory ${ }^{4}$ ), which open a way of developing powerful Web-based applications (MateCAT ${ }^{5}$ ). Many tools today even offer different versions according to users' preferences. For instance, Worfast ${ }^{6} \mathrm{TM}$ software was developed as an add-on to Microsoft Word through macros, and now is also offered as a standalone tool or as a Wed-based application, and the users can make a decision according to their tastes and budget. Thus, it is interesting to investigate how Web-based systems are perceived by professionals in the industry and what types of systems they mostly prefer.

\section{Questionnaire Design and Implementation}

The questionnaire was designed using SurveyMonkey ${ }^{7}$, an online questionnaire building tool. It was composed of separate sections, where the first section concerns the user profile, the second section includes general questions on the use of technologies, and the rest of the sections are focused on specific types of tools. This structure was chosen in order to be able to use the 'skip logic': if respondents were not familiar with tools of a particular type or were not using them in their work, most of the questions in the corresponding section were irrelevant to them, so they could be skipped automatically and the respondents were directed to the next section of the questionnaire. 'Skip logic' makes the survey navigation much easier and allows saving respondents' time and increasing the response and completion rates. The questionnaire focuses mostly on machine translation, translation memories, corpora compilation and terminology extraction, which are the main topics of research in the EXPERT project, and also covers some aspects related to quality assurance tools and various web-based lexicographical resources.

One of the main difficulties one encounters when collecting information on user requirements is the high subjectivity of obtained data. Often users are not certain about their own needs or do not know how to explain them in a clear straightforward way. In

\footnotetext{
${ }^{1}$ http://www.sdl.com/products/sdl-multiterm/desktop.html

${ }^{2}$ http://www.sdl.com/products/sdl-trados-studio/

${ }^{3}$ https://www.memoq.com/

${ }^{4} \mathrm{http}: / /$ mymemory.translated.net/

${ }^{5}$ https://www.matecat.com/

${ }^{6}$ http://www.wordfast.net/

${ }^{7}$ https://www.surveymonkey.com/

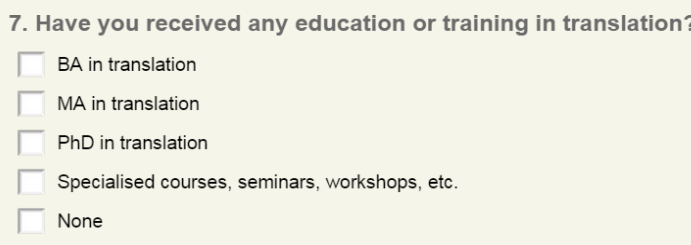

Figure 1. Question with check-boxes. 
addition, questionnaire method of collecting user information is prone to ambiguities and misunderstanding. In order to prevent this kind of issues, we carried out various preparation and testing steps prior to launching the survey. The first step consisted in analysing publicly available information, such as translators' blogs, forums, social networks and web sites that could throw light upon the most discussed topics related to translators' use of translation technologies and identify potential issues and problems that needed to be tackled. Based on this information together with various user surveys previously conducted in this field, the first draft of the questionnaire was developed. Subsequently, we proceeded to carrying out cognitive interviews with two potential respondents who worked as freelance translators. Cognitive interview is a common survey testing technique where the respondents read the questions and have to speak aloud commenting their reasoning during question answering. This way the interviewer can detect difficulties that participants might encounter while completing the survey and make sure that participants do not misinterpret any question and that the procedure of completing the survey is clear (Willis, 2005). Concurrently, we asked several representatives of the EXPERT commercial partners to complete the survey and provide feedback in terms of the questionnaire content, structure, design, and question wording. This testing stage relies on the knowledge and expertise of the professionals in the translation domain and its main purpose is to reveal more profound content-related defects of the questionnaire and possible terminological issues. After the feedback was collected both from the interviewees and the domain experts, the appropriate changes were made, and we proceeded to the last testing step, the pilot study, which consisted in collecting a small sample of responses (in our case 12) and analyse the results to identify possible defects and redundancies. After that, the final amendments were made. Other methods for avoiding ambiguity, redundancy and other problems were applied, such as 1) using as less technical jargon and very specific terms as possible; 2) using check-boxes question types, where respondents are able to select multiple options (Figure 1); 3) providing "I don't know" and "other" options for cases when the respondent does not find the most suitable answer among the ones available; 4) providing comment fields and open-ended question, where participants could answer questions in a free manner and use wording of their own choice (Figure 2).

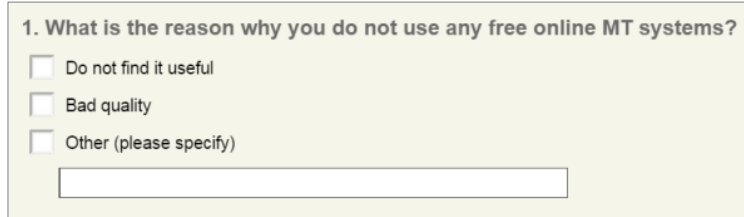

Figure 2. Question with a comment text box.

The questionnaire link was distributed through translation companies within the EXPERT project, mailing lists and social media groups for translators, translation blogs and translation associations. The participants responded actively and many provided feedback and comments. 


\section{Participants and their Profile}

We received 736 completed responses and 1304 responses in total. This indicates a high response rate but a low completion rate, which is mainly due to the large size of the questionnaire. The respondents originated from 88 different countries, about a half of them being from Italy, Spain, Germany, USA, UK, Brazil, Belgium, Finland and Portugal.

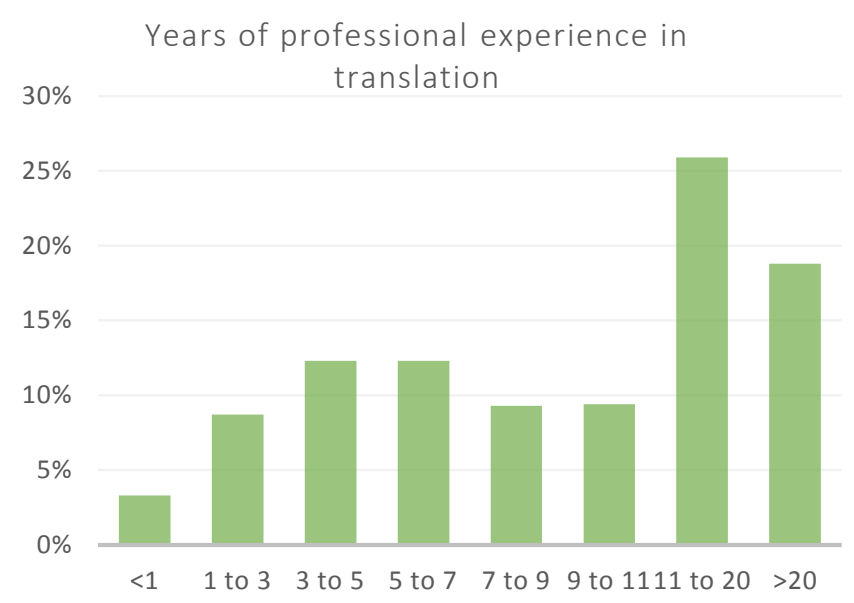

Figure 3. Years of professional experience
The majority of respondents were experienced translators and almost a half of them had more than 11 years in the industry (Figure 3 ). This is a positive fact for this research study, as most participants have good knowledge of industry and technologies available and can make judgments based on this experience.

The vast majority of translators worked freelance. Figure 4 shows the distribution of various occupation types within the sample. From the 720 respondents to this question, the two largest groups were freelancers who had an agency but also worked independently apart, and freelancers who only worked independently. Only 86 translators just worked with an agency. Other respondents worked as in-house translators in a translation company (21) or in a nontranslation company (23). Finally, 8 translators worked in a government or public institution and 10 were students. Additional 36 answers were received from translation teachers, translation company managers and CEOs, and translators working with multiple agencies. An interesting finding related to these statistics is that even though the survey was distributed through various translation service providers who sent it out

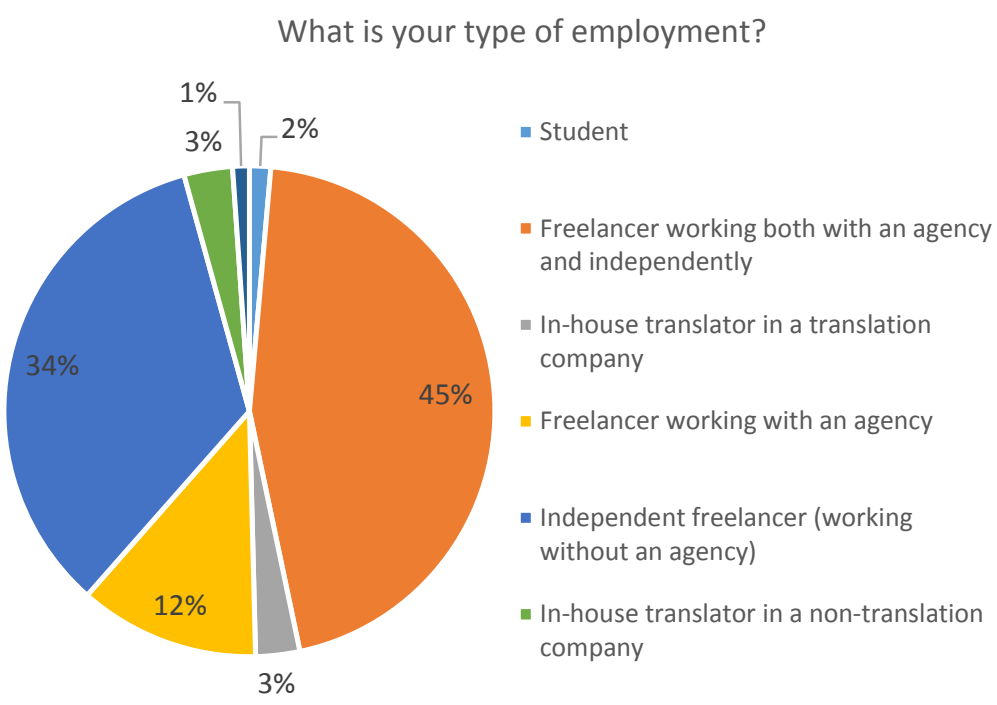

Figure 4. Type of employment 
to their freelance translators, there is still a very large number of fully independent translators. These numbers point to translators' tendency to work independently from agencies. This specific property of the sample is possibly related to the large amount of professional experience of the respondents, since many experienced freelance translators tend switch to independent way of working after gaining enough experience with a company or agency.

Among the domains of specialisation the most popular ones were, as could be expected, technical, legal, tourism and finance (Figure 5). In this question, respondents were able to choose more than just one answer and it turned out that, indeed, many of them preferred to work in more than only one domain, with the average number of domains chosen by one participant accounting to 5.16. This seems logical, since human beings tend to prefer work that involves diversity and variation rather than constant repetition, so not many translators find it satisfying to work within one domain for large periods of time.

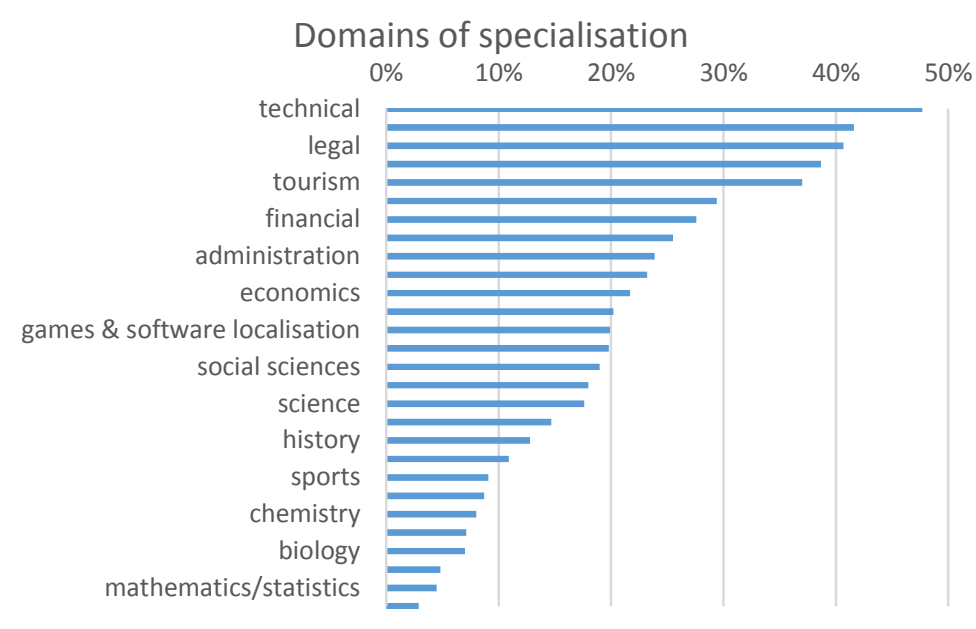

Figure 5. Domains of specialisation
Another characteristic of user profile is the education. Participants were asked whether they had received any training in the fields of translation or information technologies and computing (Tables 1 and 2). The results show that most translators had at least some education in

both fields, which can be seen as a good sign for the industry, as it means that most translators are qualified professionals. Almost a half of the sample had a university degree in translation, and $44 \%$ attended specialised courses or seminars. Surprisingly, about a quarter of respondents did not have any training in translation at all. Another significant finding is that $43 \%$ of respondents had attended courses and seminars on IT, which shows that translators have great interest in technologies and are motivated to learn how to leverage the variety of available tools and incorporate them in their workflow in a beneficial manner. Moreover, almost all participants were active computer users and evaluated their IT competence level as advanced (47\%) or experienced (42\%), while only $10 \%$ considered their level average and $1 \%$ poor.

\begin{tabular}{|l|r|}
\hline BA in translation & $\mathbf{2 1 , 4 \%}$ \\
\hline MA in translation & $\mathbf{2 5 , 0} \%$ \\
\hline PhD in translation & $\mathbf{2 , 7} \%$ \\
\hline Specialised courses, seminars, & $\mathbf{4 4 , 2} \%$ \\
\hline
\end{tabular}




\begin{tabular}{|l|l|}
\hline workshops, etc. & \\
\hline None & $\mathbf{2 4 , 6 \%}$ \\
\hline
\end{tabular}

Table 1. Training in translation

\begin{tabular}{|l|r|}
\hline BSc in IT & $3.84 \%$ \\
\hline MSc in IT & $3.56 \%$ \\
\hline PhD in IT & $0.68 \%$ \\
\hline $\begin{array}{l}\text { Specialised courses, seminars, } \\
\text { workshops on IT }\end{array}$ & $42.88 \%$ \\
\hline Specialised courses on CAT tools & $29.45 \%$ \\
\hline None & $38.90 \%$ \\
\hline
\end{tabular}

Table 2. Training in IT and computing

\section{Use of tools}

In this section we discuss how much different kinds of tools are adopted by translators, how well they have become part of the translation workflow, how regularly they use them, and why some tools are more popular than others.

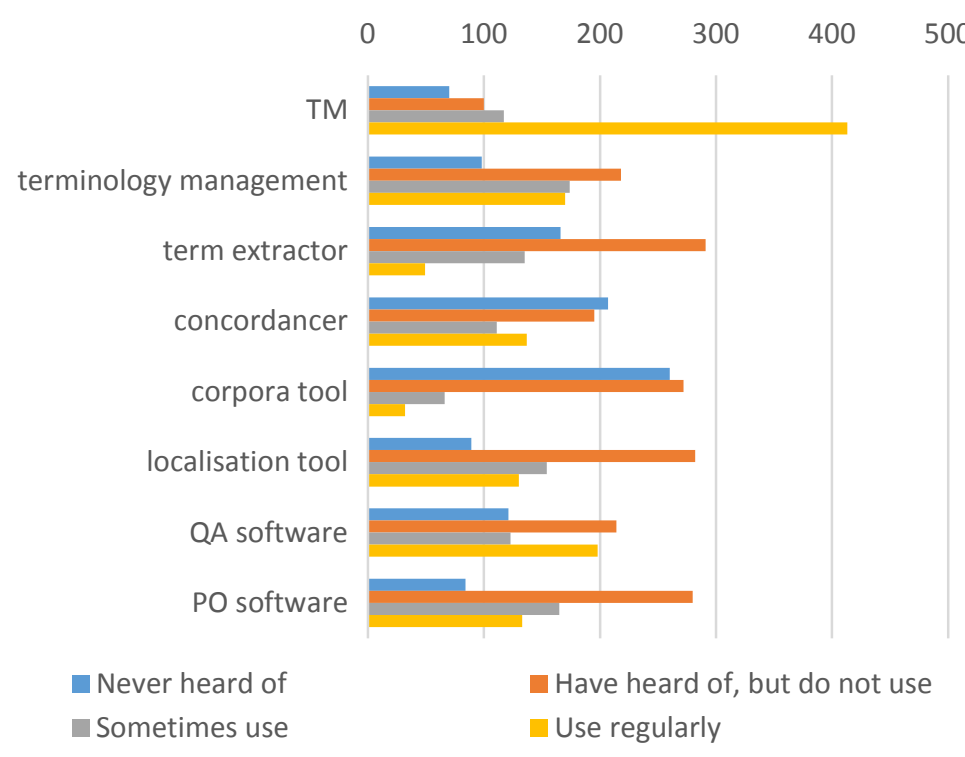

Figure 6. Familiarity with different types of tools
First of all, in order to obtain a general picture, participants were asked about their familiarity with different types of translation technologies, whether they used them on a regular basis, sometimes, were familiar but did not use them, or had never heard of them before (Figure 6). Translation memory (TM) systems appear to be the only type of tools that was used

regularly by the majority (417) of respondents to this question, while much less translators have not incorporated TM in their daily routine, but use it sometimes (113). However, there is still a considerable number of respondents who have never heard of such technology (70) and who are familiar with it but still remain reluctant to use it (100). The situation appears completely different with the other tools included in the question. Most of them were familiar to the respondents, but were not fully adopted. Only one type of technologies, concordance systems, is completely unknown to the majority of translators. Tools for compiling or managing corpora are the least common to use on a regular basis (only 32 participants), even though 242 respondents reported that 
they had heard about them before. In total, this result indicates a low rate of familiarity with less popular types of technologies, as from the 718 who answered this question, none of the tools except for TM is familiar even to the half. One of the reasons is possibly that some of the technologies mentioned in the question are now often integrated in CAT tools (as, for instance, terminology management and quality assessment). This is also confirmed by some of the comments to this question, which say that "translation software includes most of the above". Other technologies are quite specific and probably translators do not consider them appropriate for everyday use (such as tools for compiling and managing corpora). Apart from the listed tools respondents were asked to indicate any additional types of tools they use, which were aligners, OCR software, subtitle translation tools and speech recognition tools.

Do you use any machine translation software or service?

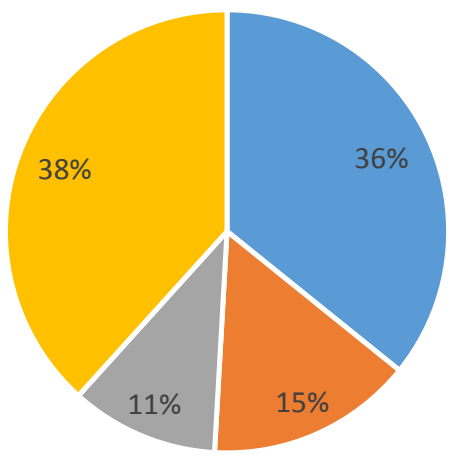

- I am currently using MT

- I am currently not using MT, but planning to use it in the future

- I used it before, but do not use it anymore

- I do not use MT and do not plan to do so in the future

Figure 7. Use of machine translation
In further sections of the questionnaire we investigated more in detail how much these and other technologies are adopted by translators and the reasons why they do or do not use them. Machine translation (MT) systems were used by $36 \%$ of respondents, while almost the same number (38\%) were not

using any MT and were not planning to use them in the future. A smaller percentage (15\%) did not reject this technology completely claiming that they were planning to use it in the future, and $11 \%$ used it before but abandoned it afterwards (Figure 7).

Several questions in the survey aimed at identifying what translators thought about MT services available online for general public. From all MT users, only 78 reported that they did not use any free online MT system, which is mostly due to bad quality (67\%) and because they did not find it useful (35\%). In addition, many respondents commented that they were not allowed to use MT due to the client's requirements. This is a common practice in the industry, as many clients are concerned about the confidentiality of their

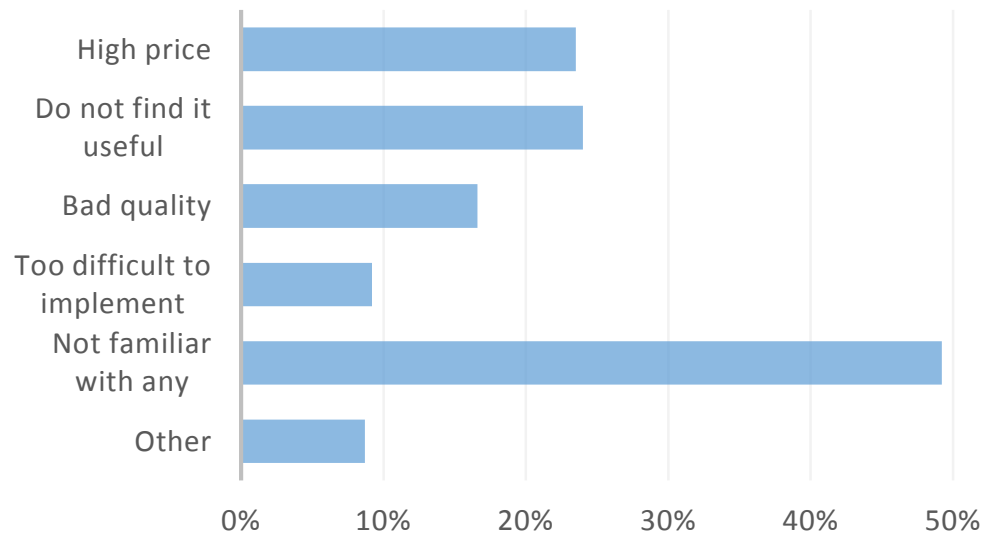

Figure 8. Reasons for not using stantalone installable 
documents and ask translators to sign an agreement on not using any online MT service.

There was a relatively small number of users who preferred working with installable MT systems. Only about $13 \%$ of MT users reported having such software installed on their computer. The most common obstacle for using installable MT was the lack of awareness of such systems and also, but less commonly, their high price, bad quality of translation, difficulty of implementation, or translators simply did not find them useful (Figure 8). Among the comments regarding this question some interesting details were discovered, such as that many respondents prefer to use the MT system integrated in their CAT tool, and in case they do not have one, they prefer to resort to online MT services.

Finally, MT users were asked if they actually had an MT system integrated in their translation software, and the answers are almost equally divided between "yes" (159), "no" (160) and “I don't know" (128).

It is not a surprise that, in comparison with automatic translation, translation memories turned out to be much more popular. Specifically, about $76 \%$ of respondents claimed that they used TM (Figure 9). Many of the TM users (73\%) said that they worked with TM by their own choice, while about a half also said that it is requested by their client and $22 \%$ were requested to use TM by their employer. This means that most of the TM users who have adopted this technology do see its benefits and use it voluntarily for their own profit.

The majority of participants (85\%) have never worked with Do you use TM systems? textual corpora (Figure 10). About a half of all the

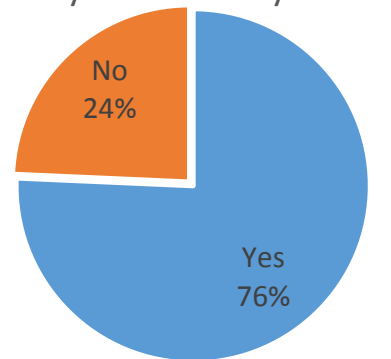

Figure 9. Use of TM systems. respondents who have worked with corpora (111) also reported that they compiled their own corpora, and only 19 of them do it with the help of special software. Translators who do not compile corpora mostly refuse to do so because it requires too much effort, as was reported by $57 \%, 40 \%$ are satisfied with the publicly available corpora, and $34 \%$ do not know about any technique for compiling corpora (Figure 11). A rather positive finding is that only $15 \%$ thought that it was not a useful thing to do, which means that most of the translators would create their own corpora if they had better methods and tools available at hand. Among the 58 respondents who did compile their own corpora,

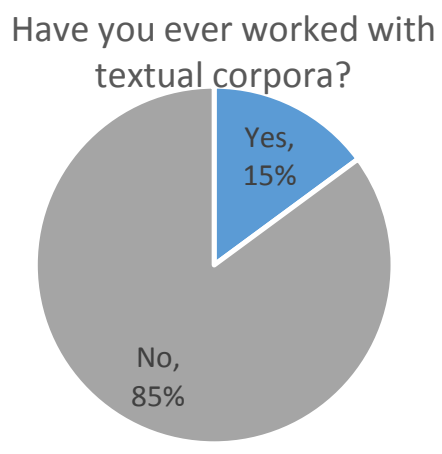
19 (about one third) resorted to some software and 39 did it manually.

Figure 10. Users working with 


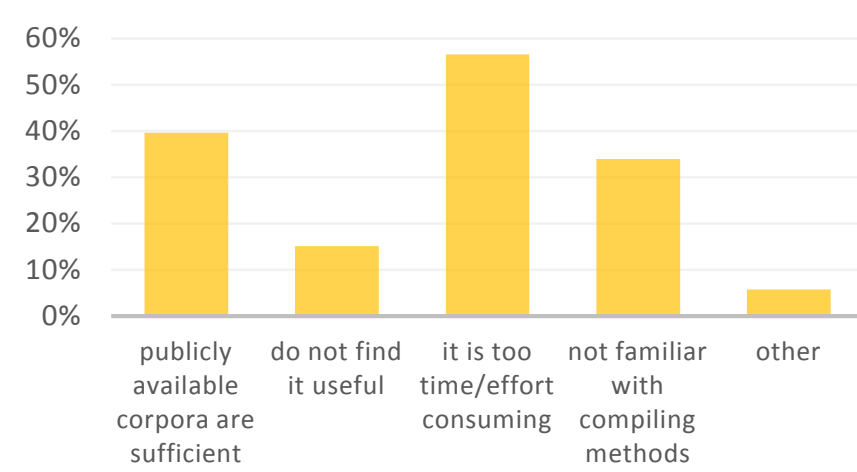

Figure 11. Reasons for not compiling

(TE) tools, on the other hand, are much less adopted among professional translators, as 75\% did not use them at all, $21 \%$ had a TE system integrated within a CAT tool, and only $4 \%$ had a standalone TE system.

Finally, the last type of tools investigated in the questionnaire was the quality assurance (QA) tools. They were reported to be used by $60 \%$ of translators, of which $35 \%$ had it integrated in their translation software, $11 \%$ had a standalone QA tool, and $14 \%$ used both type of systems.
About a half $(52 \%)$ of the translators who participated in the survey used a terminology management tool integrated in their translation software, a slightly smaller number of translators did not use any (42\%), and only $6 \%$ used a standalone terminology management tool (Figure 12). Terminology extraction

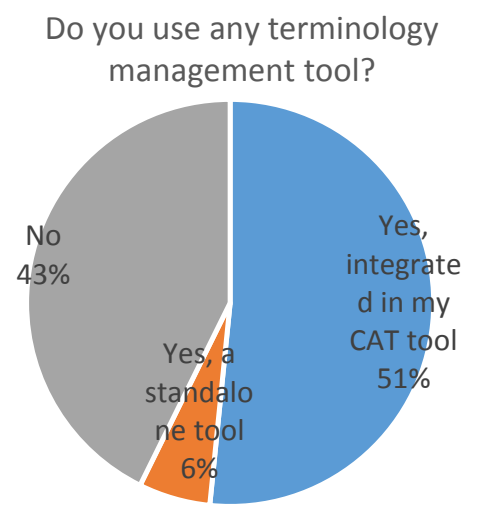

Figure 12. The use of terminology management tools.

\begin{tabular}{|c|c|c|}
\hline Type of tool & Percentage of users & Percentage of non-users \\
\hline TM & $76 \%$ & $24 \%$ \\
\hline MT & $36 \%$ & $\begin{array}{l}64 \% \text { (including } 11 \% \text { past users and } 15 \% \\
\text { future users) }\end{array}$ \\
\hline $\begin{array}{l}\text { Standalone } \\
\text { MT }\end{array}$ & $13 \%$ (of MT users) & $87 \%$ (of MT users) \\
\hline $\begin{array}{l}\text { Integrated } \\
\text { MT }\end{array}$ & $35.5 \%$ & $35.5 \%$ \\
\hline Corpora & $15 \%$ & $85 \%$ \\
\hline $\begin{array}{l}\text { Corpora } \\
\text { tools }\end{array}$ & $\begin{array}{l}17 \% \text { (of all corpora } \\
\text { users) }\end{array}$ & $83 \%$ (of all corpora users) \\
\hline $\begin{array}{l}\text { Terminology } \\
\text { management } \\
\text { tools }\end{array}$ & $58 \%$ & $42 \%$ \\
\hline $\begin{array}{l}\text { Terminology } \\
\text { extraction } \\
\text { tools }\end{array}$ & $25 \%$ & $75 \%$ \\
\hline $\begin{array}{l}\text { Quality } \\
\text { assurance }\end{array}$ & $60 \%$ & $40 \%$ \\
\hline
\end{tabular}

Table 3. Use of different types of technologies 
Table 3 puts together all the statistics collected regarding the usage of different types of technologies. It is not a big surprise that TM systems are the most widely used type, and it is rather remarkable that there is less need for machine translation than for terminology management and QA tools.

\section{Machine Translation: Practices and Attitudes}

This section reveals some of the key findings on MT, the degree of satisfaction with currently available systems and the ways they are incorporated in the translation workflow.

Please, choose the FREE ONLINE MT systems that you use (if any)

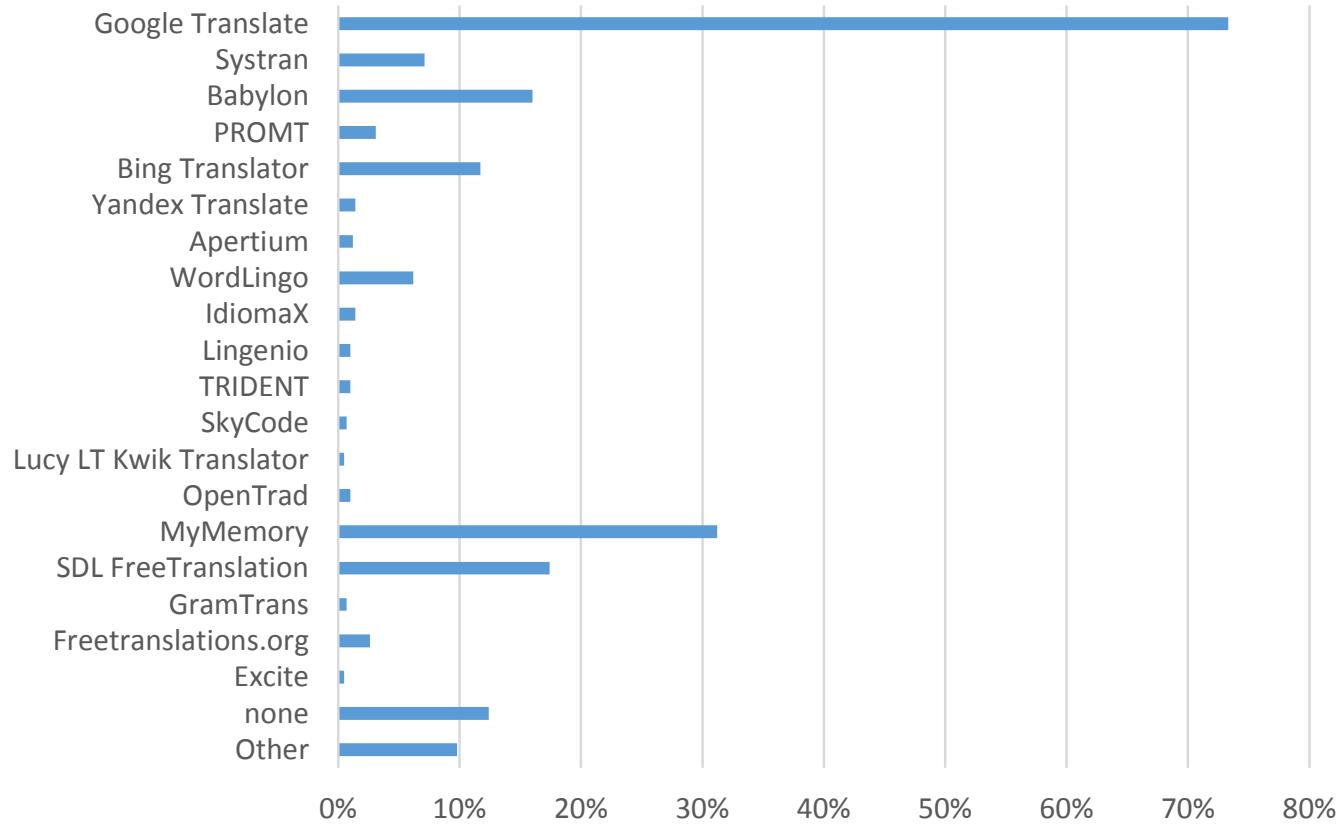

Figure 13. Use of free online MT systems

Figure 13 shows the free online machine translation systems used by the respondents. Google Translate exceeds by far all the other MT systems with $73,3 \%$ of all MT users having chosen Google Translate as their mostly used system.

However, when asked about the preferred MT system architecture, the many participants surprisingly chose hybrid MT (17\%), 12\% replied with example-based MT, only $10 \%$ chose statistical MT and rule-based accounted to $5 \%$. The majority, however, chose the "I don't know" option. The reason for this might be that most of the participants in the sample are not familiar with existing types of MT systems, but those who are have better opinions about hybrid machine translation compared to other types because they think that a rule-based component necessarily implies increased quality, which is not always the case. In addition, some translators might think that a system 
with any kind of linguistic analysis can be called hybrid, and therefore they chose the 'hybrid' option instead of 'statistical'.

In order to find out how the quality of MT translation is perceived, MT users were asked how much of correcting work they have to perform on an MT-translated text, or what percentage of words in the MT output contain errors. The results (Figure 14) show that most translators normally correct from 30 to $90 \%$ of their MT output, and the biggest number of translators ( $30 \%$ of all MT users) correct 50 to $70 \%$ of the words in the text.

These numbers confirm the fact that MT quality is still being an issue for most translators. Nevertheless, about $7 \%$ reported correcting less than $10 \%$ of MT output. In the next steps of data analysis it will be interesting to investigate how these numbers are related to the users' specialisation domain (i.e. if low correcting rate is more common for domains with higher repetition) and to their preferred MT system.

On average, how much of the MT-translated text do you usually have to correct? Choose the approximate percentage of words that have errors.

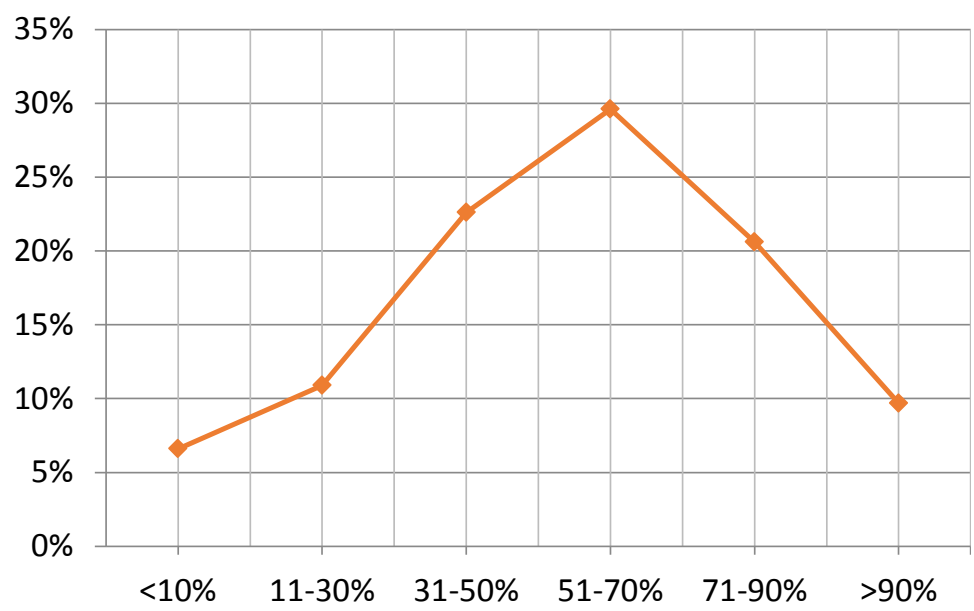

Figure 14. The amount of MT output to be corrected

It was of interest to us to find out about attitudes towards advancements in machine translation, since there is a common opinion that professional translators perceive MT as a threat for their role in the industry and a factor leading to decrease of the average hourly rate. However, the result contradicts this idea. We asked the participants if they thought they could benefit from a high quality MT system (in other words, an MT system that would translate correctly almost everything), and $74 \%$ replied "yes" compared to $26 \%$ who were
As for the motives for using MT, $58 \%$ of users resort to with the purpose of making a draft translation for further editing, $18 \%$ use MT to get the gist of the text, and a surprisingly high number (13\%) expect to obtain high quality translation (Figure 15). A significant number of respondents indicated in the comment field that they are sometimes required to use MT by their employer or agency.

What is the main purpose of using MT for you?

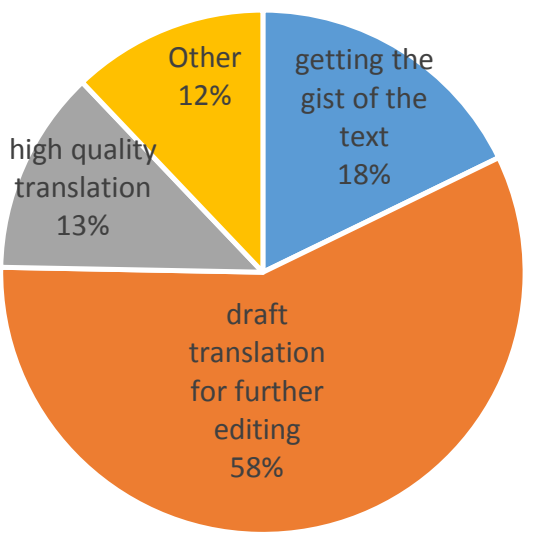

Figure 15. Purpose of MT 
against high quality MT. Among the comments provided by respondents of this question there are many positive ones, mostly mentioning "faster delivery", "shorter turnarounds", "working time and effort saving". The negative comments are mostly based on two statements. First, "a machine can never be a human", or in other words, MT will never achieve the level of translation quality that would be able to replace human translation, and it can only be a "translation support tool". The other important reason for being cautious about high quality $\mathrm{MT}$, according to the comments, is that it can jeopardize translators' way of work, and "could lead to the end of human translation in its traditional sense", and even that "companies would stop hiring translators".

Since the majority of translators in the sample still agree that MT systems could be helpful for human translators, and many of them have already adopted MT as a part of their working process, the future research should explore more thoroughly whether the translators would agree to provide the data that they generate, for instance, during postediting, in order to improve the MT systems. Some of them could prefer not to share their corrections with other translators. In addition to that, confidentiality issues may arise in relation to post-edited data use.

Only 13\% (59) of all MT users reported working with standalone installable MT software. Most commonly, respondents were not familiar with any of such tools, but other also mentioned high price, lack of usefulness, difficulty to implement, and bad quality of translation (Figure 16).

Further we wanted to find out about the use of MT integrated in translation software, and $35 \%$ reported having an MT feature in their CAT tool, while $29 \%$ did not have it. Surprisingly, a big part of respondents (36\%)

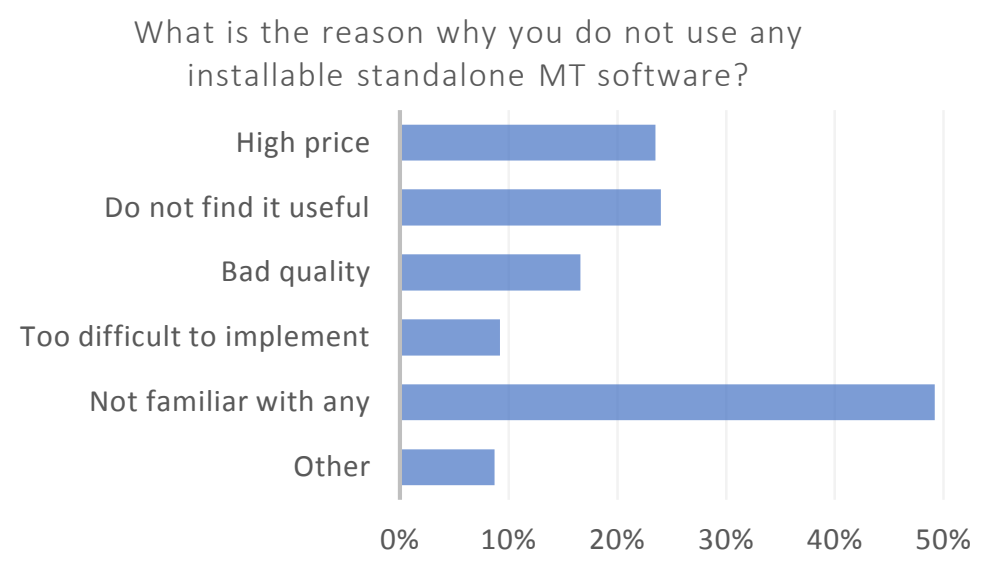

Figure 16. Reasons for not using standalone installable MT said that they did not know whether there is an MT system integrated in their CAT tool (Figure 17). This fact makes us assume that there are a number of users who are not aware of MT integration in their software, which is, consequently, one of the factors preventing them from adopting this technology in the most convenient and useful way. It is also a potential way of improving the user attitudes towards MT technology: if the users become more aware of this feature and start using it more actively, they will understand the advantages and benefit it brings. 
Do any of your CAT tools have an incorporated MT system?

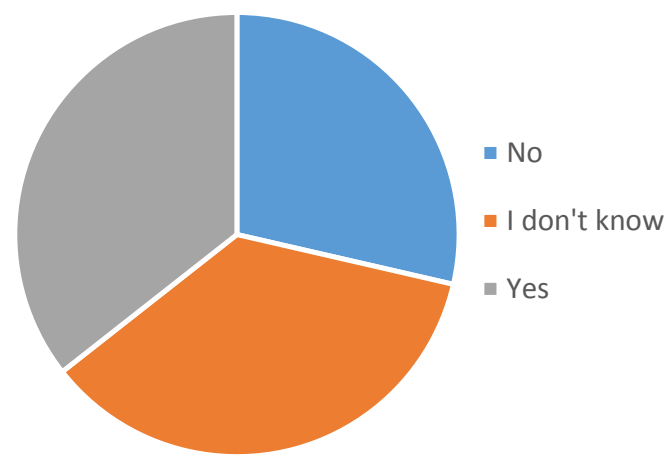

Furthermore, in general, integration of MT in translation software is perceived as something useful only by about a half of respondents (including both MT users and non-users). On a scale from 0 to 5 , where 0 is "inconvenient" and 5 is "essential", the average score received from respondents was 3.35 , with $46 \%$ viewing this feature as "useful", and $10 \%$ as "essential".

Figure 17. MT incorporated in CAT

\section{Translation Memory: Working Practices and Offered Functionalities}

The translation memory section of the questionnaire aimed specifically at identifying the features which are perceived as the most useful and helpful, features that are rarely used and even considered inconvenient, and at understanding how exactly TM software is used by professional translators.

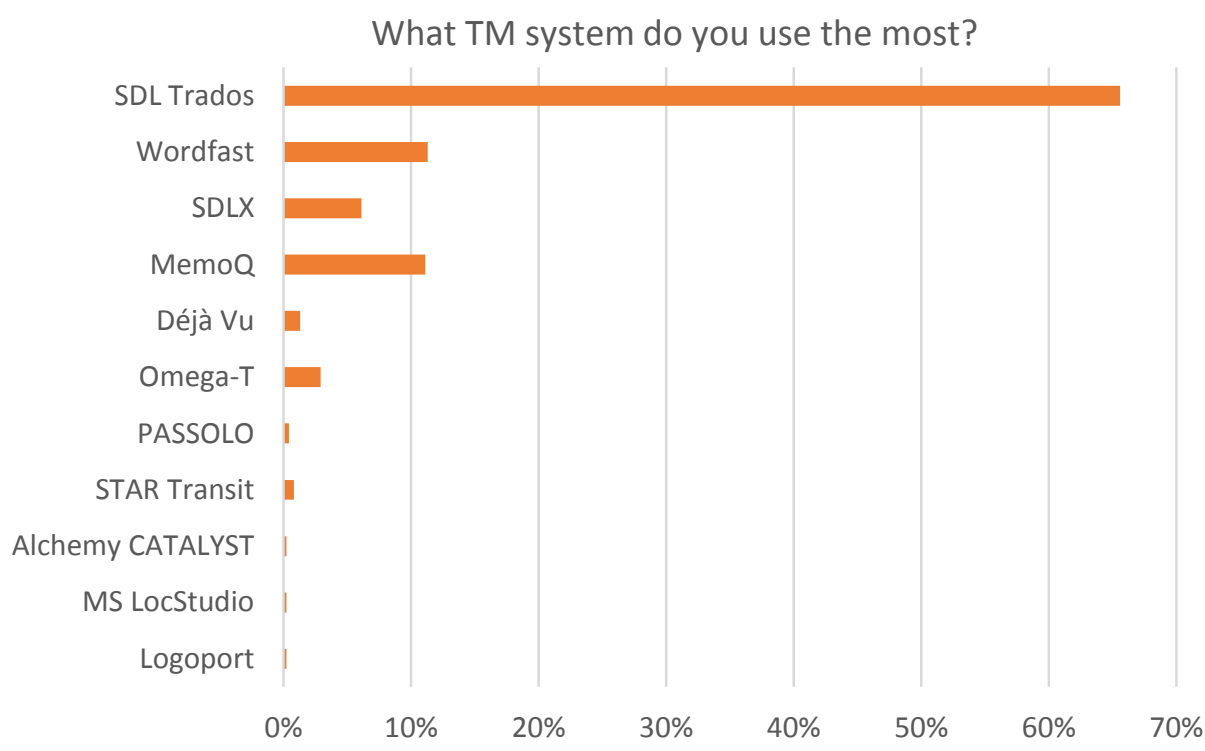

Figure 18. TM systems used by translators

Figure 18 shows the usage rate of different TM tools with SDL Trados leading by far with $65,6 \%$ of respondents. In addition, the results revealed that most translators like to work simultaneously with more than one TM (61\%), and for $42 \%$ it is normally their own TM alongside with the TM provided by the client. Some translators, however, most 
frequently work with only one TM, either their own, or the clients $(18 \%$ and $17 \%$ correspondingly), or they interchange between the two depending on the project (22\%).

Just about a half of the TM users work with more than one TM software simultaneously, of which $31 \%$ did it only rarely, while the other $19 \%$ prefer to do it more often. This might be a sign that there is no such software on the market that could satisfy everybody's needs, and many professionals have to resort to various tools to be able to take advantage of all the features they like.

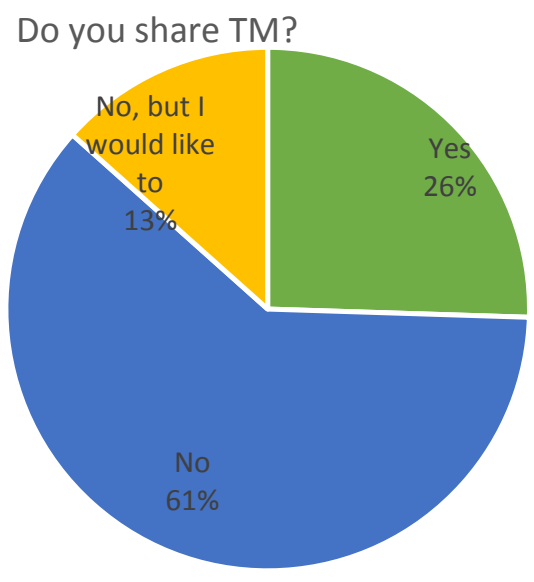

Figure 19. TM sharing

Another technology-related topic widely discussed nowadays in the context of TM is TM sharing (a specific survey on interoperability in translation industry was conducted by TAUS, 2011). In this relation $61 \%$ reported that they were not sharing TM, 26\% were doing so, and $13 \%$ were not sharing TM at that time, but they would like to (Figure 19). In the next question we asked whether translators used publicly available TM repositories, and $36 \%$ replied that they did, 32\% did not know about

this kind of resource, and the remaining $37 \%$ were aware of them but did not use them. Another question related to TM practices was where translators store their TM databases. The vast majority kept them on their computer (89\%), quite a small number of respondents (16\%) stored their TM in the cloud, and only $15 \%$ on the company's server.

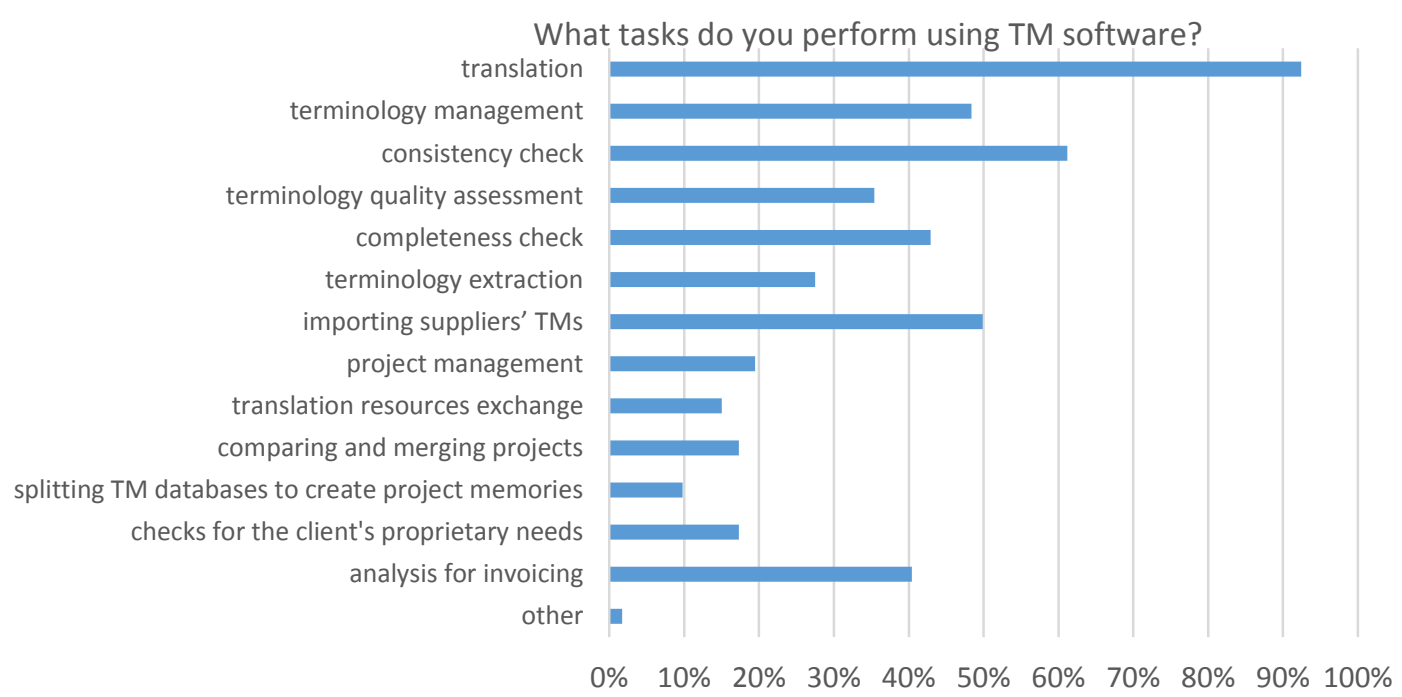

Figure 20. Tasks performed with TM software 
In order to find out more about the way TM software is used and establish possible functionalities that might turn out useful for translators, we asked them what tasks they performed with the help of their TM software. Apart from translation, it was, first of all, consistency check (reported by $61 \%$ of respondents), import of TM (50\%), terminology management (48\%), completeness check (43\%), analysis for invoicing (40\%), terminology quality assessment (35\%), terminology extraction (25\%), among others. Figure 20 includes the full list of tasks carried out with the help of TM software and the corresponding statistics.

Figure 21 illustrates how translators fill in their TMs. The most popular way to do so is during the translation (72\%), and by importing already existing the client's TM $(67,5 \%)$ or the company's TM (45\%). Some translators also align legacy translations and their originals, use parallel corpora, download publicly available TMs, among other methods.

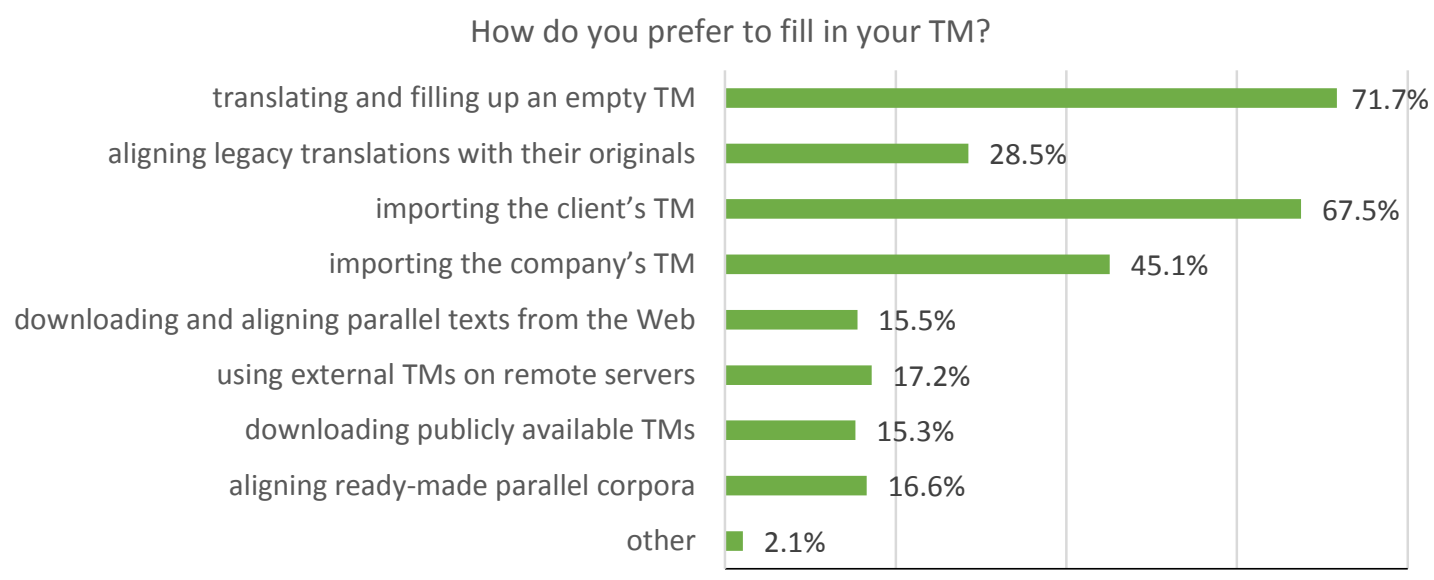

Figure 21. How translators fill in their TM

Another aspect of working with TM is that some tools only allow to edit text within the software environment, while other tools, like Wordfast, have a word processor add-on version that allows users to edit text inside their word processing software. It turned out that $18 \%$ of translators prefer to be able to use their TM in the word processor, while the majority (63\%) like to work in their TM software and $21 \%$ did not have any preference.

In another question participants had to evaluate different functionalities of TM software. They were given a list of functionalities that are contained in today's tools, and had to label them as 'essential', 'useful', 'not so useful', 'not important', or 'inconvenient'. The results of this question are illustrated in Figure 22. There is a number of features that are considered essential by many users, such as integrated terminology management system, possibility to save TM on your own PC, high working speed, simple interface and smooth learning curve, support for a big number of document formats, and support for formats originated from other TM software. In general, all of the mentioned features were regarded as useful, while some of them, such as storing data in the cloud, webbased version of the tool, or different OS versions seem less important for respondents. Additionally, many users mentioned the auto-propagation feature, which allows to 
include translated segments of the text in the TM automatically while working on one text so that they can be used later throughout the whole text.

Finally, the average time spent to learn the basics of a TM program accounted to 28 hours. A positive result is that the majority of respondents considered the advantages of using TM software worth the time spent on training, with $72 \%$ saying that it was "definitely worth it", and $23 \%$ with "probably worth it".

\section{Textual Corpora and Related Tools}

In the section about textual corpora we tried to find out what types of corpora are used the most. Among the 110 translators who used corpora in their work, 48\% preferred free publicly available corpora, $45 \%$ used Web as a corpus, and $41 \%$ used online corpora of web texts. Some respondents compiled their own ad hoc corpora (35\%), 26\% used online corpora with concordancers, $22 \%$ compiled their own corpora for general purposes (instead of creating a separate corpus for each project), and $8 \%$ used commercial corpora (Figure 24). 
Another way of classifying corpora is with respect to their content (i.e. comparable or non-comparable corpora) and languages (monolingual, bilingual, parallel). For instance,

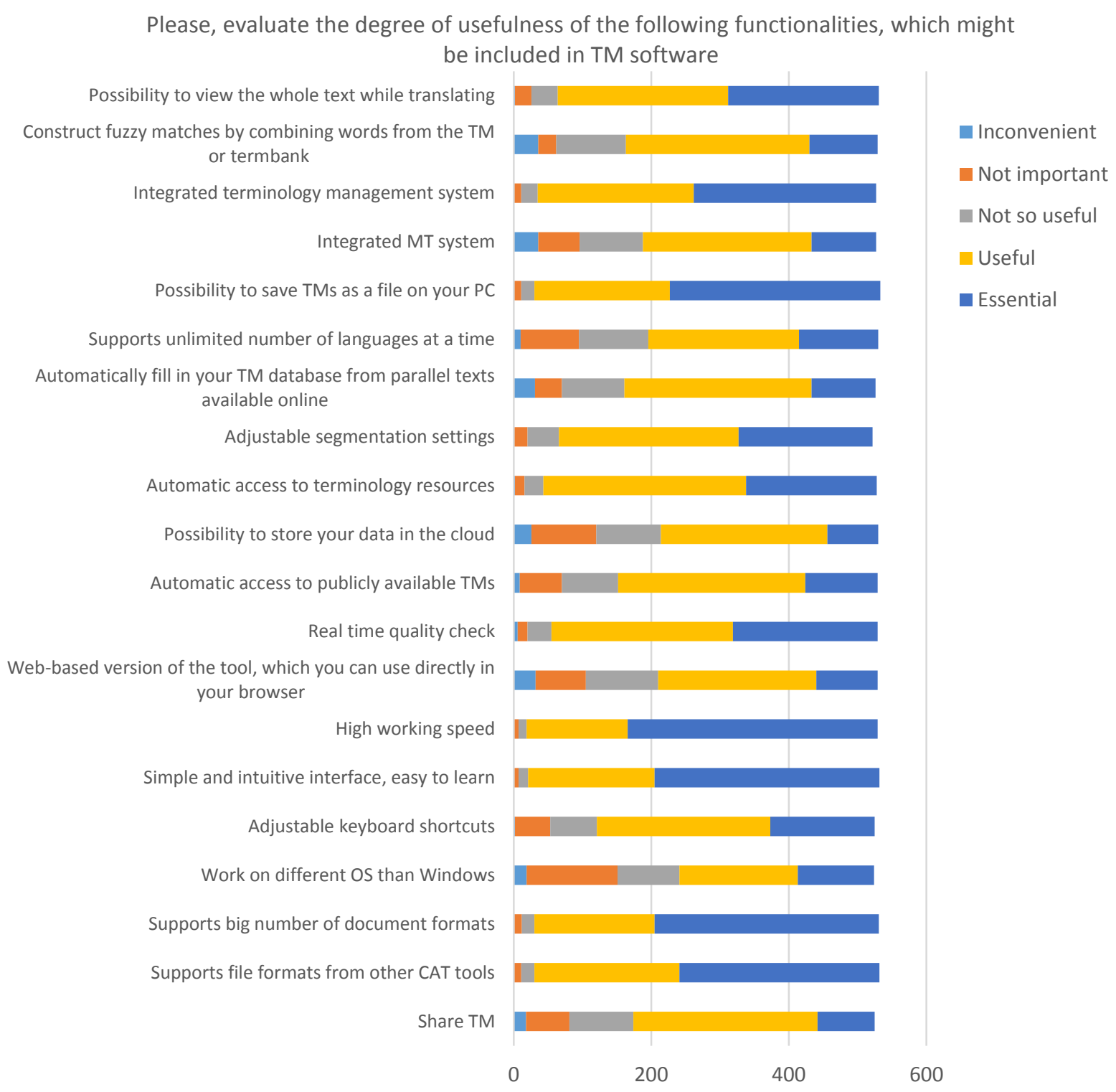

Figure 22. Preferred features of TM software

the most popular type turned out to be bilingual parallel corpora (collections of texts and their translations into another language), which were used by $67 \%$. Monolingual corpora were used by $43 \%$ of total corpora users, and $40 \%$ reported using multilingual parallel corpora. Comparable corpora (collections of texts on relates subjects) were used only by $22 \%$ (Table 4 ). The fact that translators prefer parallel corpora to comparable corpora is not a surprise. For translation, it is more practical to search a parallel corpora available online and retrieve a sentence that contains a good translation for the source segment. However, for restricted domains parallel corpora are often not available, and translators do not have time and resources to build a parallel corpus from scratch. On the other hand, comparable corpora are easier to compile, and they are useful for translators, but they have not adopted this practice, as has been pointed out in one of the previous sections, mostly because they do not think that the time and effort spent 
on compilation will pay off. Therefore, simple, fast, and easy to use tools for compiling comparable corpora can be a solution. However, there does not exist a tool on the market that fits these requirements (Costa et al., 2014).

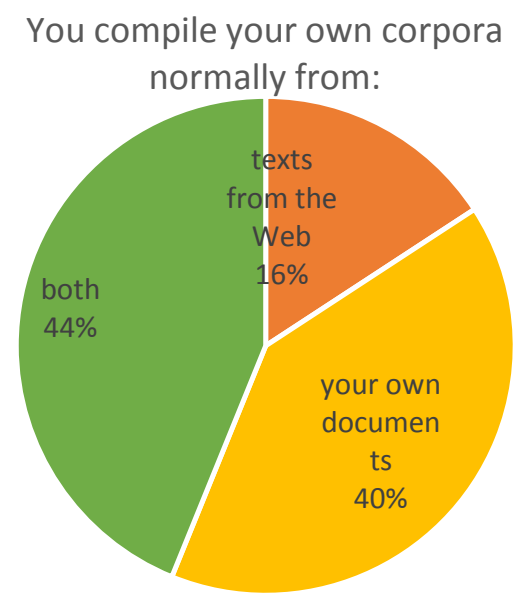

Figure 23. Texts that translators use to compile corpora

In addition, we asked what kind of texts translators normally use to compile corpora (Figure 23) and most of them (44\%) appear to combine their own documents with documents from the Web and $40 \%$ only use their own documents.

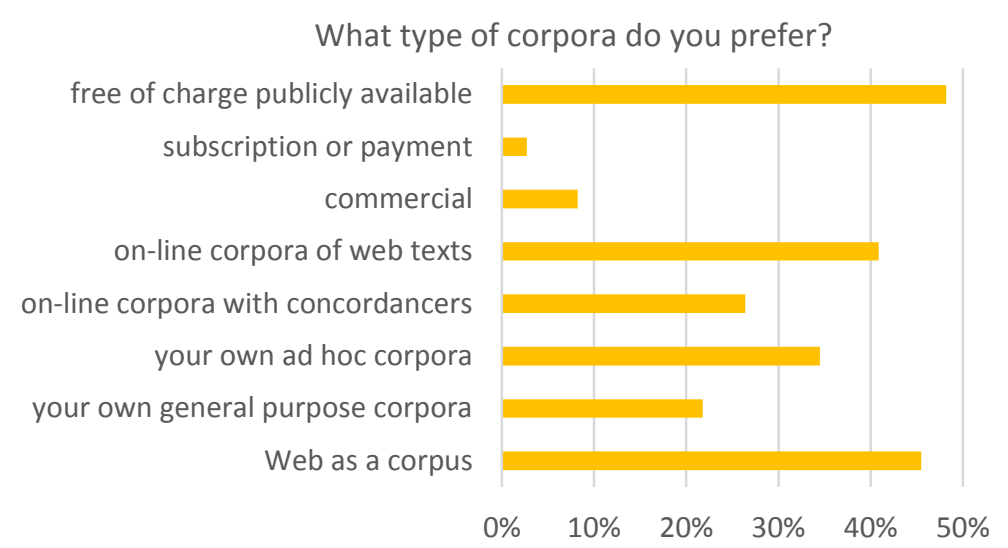

Figure 24. Prefered types of corpora
In order to find out which features are the best to be included in such tool for compiling corpora we asked respondents to evaluate some features as essential, useful, not so useful, not important, or inconvenient (Figure 25). Only 29 translators replied to this question (due to

the small total number of respondents who did compile corpora), of which 20 consider concordance an essential functionality in this type of tools. Indeed, being able to search words in context is the main purpose of using corpora for translators, especially it is so for comparable corpora, where they do not have direct translations which they can use without considering the context of the searched word or segment. Other features that were mostly considered useful or essential were simple interface, possibility to manage corpora, i.e. explore, delete, and rearrange documents into different corpora, and also to reuse old documents when building a new corpus, as well as automatic retrieval of Web documents. Part-of-speech tagging appeared to be less important, as it received the lowest 'essential' rate and the highest 'not so useful' rate. POS-tagging and terminology extraction were the only options that were inconvenient to one user. 


\begin{tabular}{|l|l|}
\hline $\begin{array}{l}\text { bilingual parallel } \\
\text { corpora }\end{array}$ & $66,7 \%$ \\
\hline $\begin{array}{l}\text { monolingual } \\
\text { corpora }\end{array}$ & $43,2 \%$ \\
\hline $\begin{array}{l}\text { multilingual } \\
\text { parallel corpora }\end{array}$ & $39,6 \%$ \\
\hline $\begin{array}{l}\text { comparable } \\
\text { corpora }\end{array}$ & $21,6 \%$ \\
\hline
\end{tabular}

Table 4. Types of corpora translators use.

Other interesting features that respondents mentioned in the comment field were "language recognition feature for false entries", "self-zip and extraction ability for PC storage", "side notes, margins or highlighting for certain words or phrases".
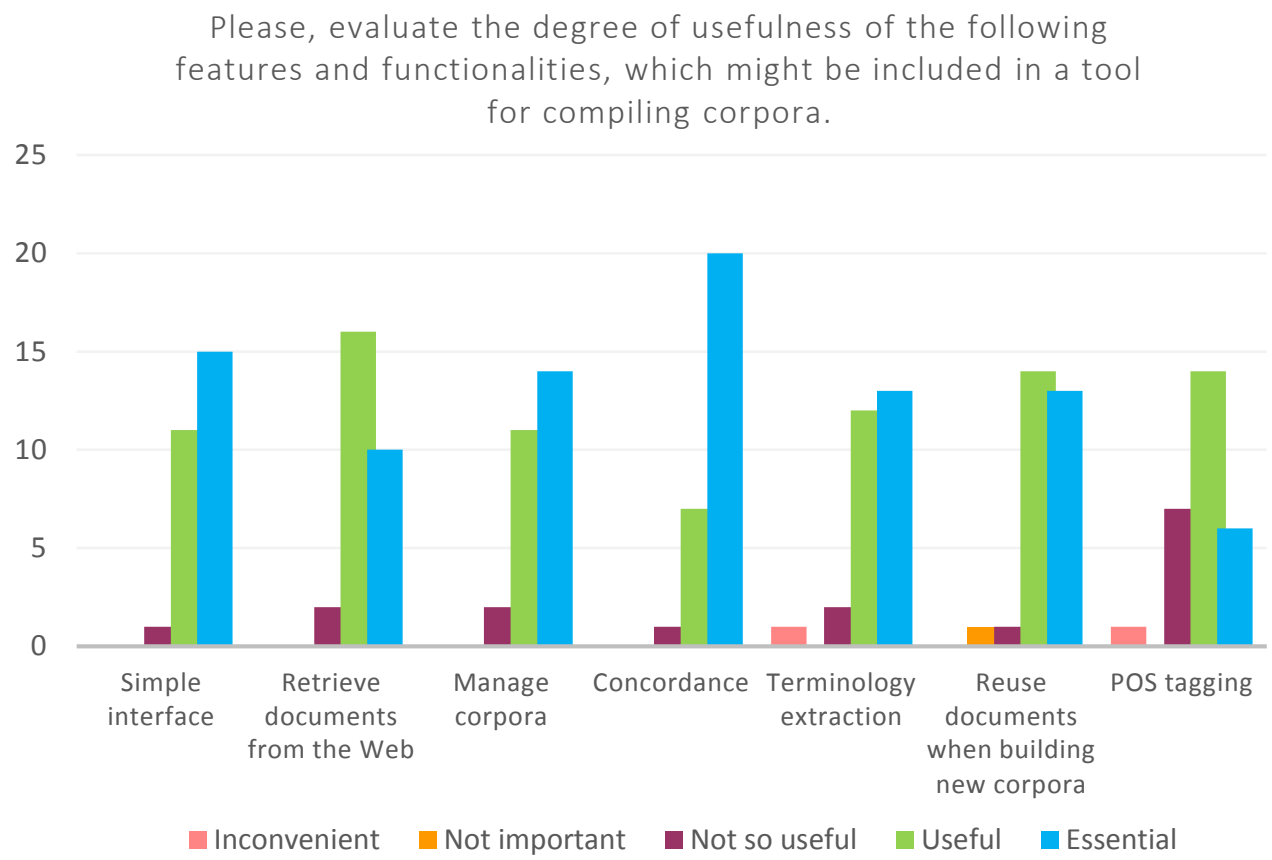

Figure 25. What features are useful in a corpora compilation tool?

\section{Conclusions}

In this report we discussed the key findings of the descriptive analysis of the data collected as a result of a survey for professional translators that intended to investigate their requirements to translation technologies. We focused on various characteristics of the user profile, how many of the respondents use such technologies as machine 
translation, translation memories, textual corpora and tools for building corpora, terminology extraction and management tools, quality assurance systems.

Most of the respondents were experiences freelance translators and had a university degree or some training in translation and a considerable number additionally had training in IT, which shows their high interest in technology.

Already after this first stage of data analysis it is possible to make some suggestions about the kind of tools professional translators actually need. The majority of translators used TM, which is no surprise as it is a common industry practice nowadays. The most important characteristics that a TM system should have, according to the respondents, are high working speed, simple interface and smooth learning curve, support for a big number of document formats including formats from other TM software, integrated terminology management system, possibility to save TM on their own PC, automatic consistency and completeness check (and quality assurance in general), and analysis for invoicing. Another feature that was mentioned by respondents was auto-propagation, which is included in the new versions of many popular TM systems. On the contrary, sharing and using open online TM repositories is still not very common as the users do not see any benefits from sharing.

Most of respondents remained reluctant to use machine translation mainly due to low quality and to confidentiality requirements imposed by their clients. Disregarding the quality issue, MT users still expressed positive attitude towards this technology arguing that it leads to faster delivery. Furthermore, the majority thought that they could benefit from high quality machine translation that would have almost $100 \%$ accuracy, which shows the quality issue is the main obstacle which prevents MT developers from reaching a wider population of users. One of the potential solutions to be investigated is implementing various techniques for user feedback collection from interaction between the user and the MT system.

Very few translators worked with corpora and even fewer compiled their own corpora using compiling software, as they found it too time consuming and were not familiar with any special tools that could make the task easier for them. It makes us assume that having an easy-to-use program for compiling and managing parallel and comparable corpora can turn out useful for translators and help include corpora in their workflow.

In the following steps of date analysis, it is planned to correlate different variables in order to discover possible underlying dependencies and relations in the collected data. For instance, we aim to answer the following questions: How do the working languages influence the use of technology? Are there gaps in the use of technologies with resource-poor languages? Are there countries where some technologies are less adopted? How do users get to know about various technologies? Is there a connection between computer training and higher usage rates? What is the relation between domain of specialisation and use of different types of tools? 
After that we will proceed to the qualitative data analysis that will consist in gathering, structuring and analyzing the information from respondents' comments and open-ended questions.

\section{References}

Alabau, V., Leiva, L. A., Ortiz-Martínez, D., \& Casacuberta, F. (2012). User Evaluation of Interactive Machine Translation Systems. EAMT 2012: Proceedings of the 16th Annual Conference of the European Association for Machine Translation, Trento, Italy, May 28302012.

Blancafort, H., Heid, U., Gornostay, T., Méchoulam, C., \& Daille, B. (2011). User-centred Views on Terminology Extraction Tools: Usage Scenarios and Integration into MT and CAT Tools. Tralogy [on-Line] Session 1 - Terminologie et Traduction.

Bowker, L., \& Corpas Pastor, G. (2014). Translation Technology. To appear in: Handbook of Computational Linguistics. (ed. R. Mitkov). 2nd ed. Oxford: Oxford University Press.

Costa, H., Corpas Pastor, G., and Seghiri, M. (2014). iCompileCorpora: A Web-based Application to Semi-automatically Compile Multilingual Comparable Corpora. In 36th Translating and the Computer Conference (London, UK, November 2014), AsLing.

Courage, C., \& Baxter, K. (2005). Understanding Your Users: A Practical Guide to User Requirements: Methods, Tools, and Techniques. Gulf Professional Publishing

Koehn, P., \& Haddow, B. (2009). Interactive Assistance to Human Translators using Statistical Machine Translation Methods. In MT Summit XII: 12th Machine Translation Summit (pp. 73-80). Ottawa, Ontario, Canada.

Lagoudaki, E. (2006). Translation Memories Survey 2006 : Users' perceptions around TM use. In Proceedings of the ASLIB International Conference Translating \& the Computer 28, London.

Langford, J., \& McDonagh, D. (2003). Focus Groups: Supporting Effective Product Development. CRC Press.

Maguire, M., \& Bevan, N. (2002). User requirements analysis. A review of supporting methods. In J. Hammond, T. Gross, \& J. Wesson (Eds.) Usability. Gaining a Competitive Edge. (pp. 133-148). Springer US.

Ortiz-Martínez, D., García-Varea, I., \& Casacuberta, F. (2010). Online Learning for Interactive Statistical Machine Translation. In HLT-2010: Conference of the North American Chapter of the Association for Computational Linguistics (pp. 546-554). Los Angeles, California.

TAUS. (2011). Translation industry interoperability http://www.w3.org/International/multilingualweb/pisa/slides/vandermeer.pdf 
Torres Domínguez, R. (2012). 2012 Use of Translation Technologies Survey. Retrieved from http://mozgorilla.com/download/19/

Willis, G. B. (2005). Cognitive interviewing: a tool for improving questionnaire design. Thousand Oaks, CA. 